\title{
High-Field EPR Spectroscopy on Transfer Proteins in Biological Action
}

\author{
K. Möbius*, A. Schnegg, M. Plato, M.R. Fuchs ${ }^{\dagger}$ \\ AND A. SAVITSKY \\ Department of Physics, Free University Berlin \\ Arnimallee 14, 14195 Berlin, Germany
}

\begin{abstract}
In the last decade joint efforts of biologists, chemists, and physicists were made to understand the dominant factors determining specificity and directionality of transmembrane transfer processes in proteins. Characteristic examples of such factors are time varying specific $\mathrm{H}$-bonding patterns and/or polarity effects of the microenvironment. In this overview, a few large paradigm biosystems are surveyed which have been explored lately in our laboratory. Taking advantage of the improved spectral and temporal resolution of high-frequency/high-field EPR at $95 \mathrm{GHz} / 3.4 \mathrm{~T}$ and $360 \mathrm{GHz} / 12.9 \mathrm{~T}$, as compared to conventional X-band EPR $(9.5 \mathrm{GHz} / 0.34 \mathrm{~T})$, three transfer proteins in action are characterized with respect to structure and dynamics: (1) light-induced electron-transfer intermediates in wild-type and mutant reaction-centre proteins from photosynthetic bacteria Rhodobacter sphaeroides, (2) light-driven proton-transfer intermediates of site-specifically nitroxide spin-labelled mutants of bacteriorhodopsin proteins from Halobacterium salinarium, (3) refolding intermediates of site-specifically nitroxide spin-labelled mutants of the channel-forming protein domain of Colicin A bacterial toxin produced in Escherichia coli. The information obtained is complementary to that of protein crystallography, solid-state NMR, infrared and optical spectroscopy techniques. A unique strength of high-field EPR is particularly noteworthy: it can provide detailed information on transient intermediates of proteins in biological action. They can be observed and characterized while staying in their working states on biologically relevant time scales.
\end{abstract}

PACS numbers: $76.30 . \mathrm{Rn}$

\footnotetext{
${ }^{*}$ corresponding author; e-mail: klaus.moebius@physik.fu-berlin.de

${ }^{\dagger}$ Present address: Proteinstrukturfabrik c/o Bessy GmbH, Albert-Einstein-Strasse 15, 12489 Berlin, Germany
} 


\section{Introduction}

Most biologists, and many chemists and physicists, are familiar with electron paramagnetic resonance (EPR) only in its conventional continuous-wave (cw) $\mathrm{X}$-band version, operating at a microwave frequency of about $9.5 \mathrm{GHz}$. In contrast to EPR, nuclear magnetic resonance (NMR) has been established over the last 20 years as a multi-frequency tool in life sciences for spectacular applications in structure determination and imaging. Not surprisingly, therefore, as many as four Nobel prizes for NMR methodology and applications have been awarded within the last 13 years (to R.R. Ernst in Chemistry in 1991, to K. Wüthrich in Chemistry in 2002, to P.C. Lauterbur and P. Mansfield in Physiology and Medicine in 2003). It is only during the last decade that the chemistry, biology, and physics communities witness a dramatic catching up of EPR because of technological breakthroughs in pulsed microwave, sweepable cryomagnet, and fast data acquisition instrumentation. In fact, modern EPR is boosting now rather similar to what had happened with NMR 10 years earlier.

The time scales of the NMR and EPR experiments are determined by the nuclear and electron resonance frequencies (in the rf and mw domains, respectively), the characteristic frequency separations in the respective spectra $(\mathrm{Hz}$ versus $\mathrm{MHz})$ and the relaxation times $T_{1}, T_{2}$ (ms versus $\mu \mathrm{s}$ ). Because of the long nuclear $T_{1}$ and $T_{2}$ times in diamagnetic molecules, NMR pulses need not be shorter than $1 \mu \mathrm{s}$, which to coherently generate and detect does not pose technical problems. The electronic relaxation times are typically in the $\mu$ s range or shorter $\left(T_{2}\right)$ and, consequently, in EPR the mw pulses have to be as short as a few ns. To generate them poses great technical problems even today in terms of mw sources and fast computers to detect and handle the transient signals in the ns time scale. Thus, the instrumental requirements for Fourier-transform (FT) EPR are much tougher to fulfil than for FT-NMR, and this explains why pulse EPR has become popular only since the late 80 's. The pioneering work in pulse EPR was done by J.H. Freed [1] at Cornell and A. Schweiger [2] at ETH Zurich and represents milestone progress in modern EPR spectroscopy.

For radicals with unpaired electron spins with $S=1 / 2$ coupled by hyperfine interaction with nuclear spins $I_{i}$ the static spin Hamiltonian, $\hat{\boldsymbol{H}}_{0}$, that describes the time-independent spin-interaction energies in an external "Zeeman" field $\boldsymbol{B}_{0}$, consists of three terms

$$
\hat{\boldsymbol{H}}_{0} / h=\frac{\mu_{\mathrm{B}}}{h} \cdot \boldsymbol{B}_{0} \cdot \tilde{g} \cdot \hat{\boldsymbol{S}}-\sum_{i} \frac{g_{n i} \mu_{K}}{h} \cdot \boldsymbol{B}_{0} \cdot \hat{\boldsymbol{I}}_{i}+\sum_{i} \hat{\boldsymbol{S}} \cdot \tilde{A}_{i} \cdot \hat{\boldsymbol{I}}_{i}
$$

i.e., the field-dependent electron and nuclear Zeeman interactions and the field-independent electron-nuclear hyperfine interactions $(h-$ Planck constant; $\mu_{\mathrm{B}}, \mu_{K}-$ Bohr and nuclear magnetons; $g_{\mathrm{n}}-$ nuclear $g$-factors; $\hat{\boldsymbol{S}}, \hat{\boldsymbol{I}}-$ electron and nuclear spin vector operators; the summation is over all nuclei). 
The interaction tensors $\tilde{g}$ and $\tilde{A}_{i}$ are probing the electronic structure (wave function) of the molecule either globally ( $\tilde{g}$ tensor) or locally (hyperfine tensors). The tensors contain isotropic and anisotropic contributions. In isotropic fluid solution, only the scalar values, $(1 / 3) \operatorname{Tr}(\tilde{g})$ and $(1 / 3) \operatorname{Tr}(\tilde{A})$, are observed. In frozen solutions, powders or single crystals, on the other hand, also anisotropic tensor contributions become observable provided appropriate resolution conditions prevail.

In the strong-field approximation, the energy eigenvalues of Eq. (1) are classified by the magnetic spin quantum numbers, $m_{S}$ and $m_{I}$, and are given, to the first order, by

$$
E_{m_{S} m_{I}} / h=\frac{g^{\prime} \mu_{\mathrm{B}}}{h} B_{0} m_{S}-\sum_{i} \frac{g_{n i} \mu_{K}}{h} B_{0} m_{I_{i}}+\sum_{i} A_{i}^{\prime} m_{S} m_{I_{i}},
$$

where the scalar quantities $g^{\prime}$ and $A^{\prime}$ contain the desired information about magnitude and orientation of the interaction tensors.

For single-crystal samples, the complete tensor information can be extracted from the angular dependence of the resonance lines when the crystal is rotated in its three symmetry planes ("rotation patterns"). Well below room temperature, the overall rotation of the protein complex becomes so slow that powder-type spectra are obtained. Nevertheless, under certain circumstances which depend on the magnitude of the anisotropy of the interactions in the spin Hamiltonian in comparison to the inhomogeneous linewidth, even from disordered powder-type EPR spectra single-crystal like information can be extracted by applying orientation-selective electron nuclear double resonance (ENDOR) [3, 4]. In the case of transition-metal complexes the hyperfine anisotropy may provide this orientation selectivity from the entire orientational distribution of the molecules [3]. In the case of organic radicals with small hyperfine interactions one has to resort to the anisotropy of the Zeeman interaction which can become large enough at high $B_{0}$ fields to provide the desired orientation selectivity for ENDOR experiments [4]. The best approach for elucidating molecular structure and orientation in detail is, of course, to study single-crystal samples. Unfortunately, to prepare them is often difficult or even impossible for large biological complexes.

\section{Why high-field EPR and ENDOR?}

From the spin Hamiltonian (Eq. (1)) one sees that some interactions are magnetic field-dependent (the Zeeman interactions), while others are not (the hyperfine interactions). Obviously, in complex biological systems it will be necessary to measure at various field/frequency settings in order to separate these interactions from each other. Up to now, cw and time-resolved (tr) EPR studies on biological samples have been concentrated on standard X-band frequencies (9.5 GHz), extensions to lower (S-band, $4 \mathrm{GHz}$ ) and higher microwave frequencies 
(K-band, $24 \mathrm{GHz}$; Q-band, $35 \mathrm{GHz}$; W-band, $95 \mathrm{GHz}$, or even higher frequencies) are exceptions.

For low-symmetry systems, particularly in frozen solution samples, standard EPR suffers from strong inhomogeneous line broadening, i.e., from low spectral resolution. Such problems arise, for instance, because several radical species or different magnetic sites of rather similar $g$-values are present or a small $g$-tensor anisotropy of the paramagnetic system does not allow canonical orientations of the powder EPR spectrum to be observed. In such a case, even X-band ENDOR may not be sufficiently orientation-selective to provide single-crystal type information of the hyperfine structure. For improving the spectral resolution by high-field EPR, a true high-field experiment must fulfil the following condition:

$$
\frac{\Delta g}{g_{\text {iso }}} B_{0}>\Delta B_{1 / 2}^{\mathrm{hf}},
$$

i.e., the anisotropic electron Zeeman interaction must exceed the inhomogeneous line broadening, $\Delta B_{1 / 2}^{\mathrm{hf}}$. For example, for deuterated samples, Q-band EPR might already fulfil this condition in the case of semiquinone radicals with rather large $g$-anisotropy, whereas for protonated samples with inherently larger linewidths, it does not. On the other hand, in the case of chlorophyll ion radicals, due to their small $g$-anisotropy, even W-band EPR might not meet the high-field condition for protonated samples. Then deuteration of the sample will be necessary or, as an alternative, to further increase the mw frequency and $B_{0}$ field, for instance by resorting to $360 \mathrm{GHz}$ EPR (see below).

Over the last 20 years a small number of dedicated laboratories has met the technological challenge to construct $\mathrm{mm}$ and sub-mm high-field EPR and ENDOR spectrometers, thereby opening a promising new research area. The physical principles and technical aspects have been published by the laboratories involved. The pioneering high-field EPR work was done by Lebedev [5] in Moscow. Appropriate references to these laboratories are included in recent overview articles, for instance [6]. Details of the laboratory-built $95 \mathrm{GHz}$ and $360 \mathrm{GHz}$ EPR/ENDOR spectrometers at FU Berlin have been published elsewhere (see Refs. [7-10]).

\section{Application of high-field EPR to paradigmatic protein systems}

In this overview we focus on a selection of protein systems that were previously crystallized and for which high-resolution X-ray structures have been made available by now: bacterial photosynthetic reaction centres (RCs) for light-induced electron transfer across the membrane; bacteriorhodopsin (BR), the light-driven transmembrane proton pump; Colicin A, the transmembrane ion-channel forming bacterial toxin. These proteins have been characterized in detail over the last years by powerful spectroscopic techniques including ultra-fast laser spectroscopy, FT-IR, solid-state NMR, and multifrequency EPR. In addition, sophisticated theoretical studies had been performed to elucidate their light-induced electron and 
proton transfer characteristics. Hence, these proteins represent paradigm systems of general interest. They are well suited for new multifrequency high-field EPR experiments to study functionally important transient states during biological action. We prefer EPR as the method of choice because of the accessibility of functionally important time windows for the transient states. These states are either directly detectable by EPR by their transient paramagnetism as, for instance, the radical-ion and radical-pair states of cofactors involved in photosynthetic electron transfer in RCs or, more indirectly, by site-specific nitroxide spin-labelling of the protein as, for instance, in site-directed mutants of BR and Colicin A. It will be shown that high-field EPR experiments allow specific protein regions to be identified and characterized as molecular switches for vectorial transfer processes across membranes. Such switches are envisaged to function by controlling the electronic structure of cofactors, for example by invoking weak cofactor-protein interactions via H-bonds, by polarity gradients or even by substantial cofactor and/or helix displacements.

The high-field EPR work on the RC, BR, and Colicin A was performed in collaboration with the research groups of W. Lubitz (Mülheim) for the RC studies and of H.-J. Steinhoff (Osnabrück) for the BR and Colicin A studies.

\subsection{Bacterial photosynthetic reaction centres of Rb. Sphaeroides}

Photosynthesis is the most important process that enables life on Earth by converting the energy of sunlight into electrochemical energy needed by higher organisms for synthesis, growth, and replication. The so-called primary processes of photosynthesis are those in which the incoming light quanta, after being harvested by "antenna" pigment/protein complexes and channelled to the $\mathrm{RC}$ complexes by ultra-fast energy transfer, initiate electron-transfer (ET) reactions between protein-bound donor and acceptor pigments across the cytoplasmic membrane. The successive charge-separating ET steps between the various redox partners in the transmembrane $\mathrm{RC}$ have very different reaction rates, $k_{\mathrm{ET}}$. The lifetimes, $t_{1 / 2}=\left(k_{\mathrm{ET}}\right)^{-1}$, of the transient charge-separated states range from less than 1 ps for neighbouring donor-acceptor pigments to more than $1 \mathrm{~ms}$ for large donor-acceptor separations on opposite sides of the membrane (ca. $40 \AA$ ). The cascade of charge-separating ET steps of primary photosynthesis competes extremely favourable with wasteful charge-recombination ET steps thereby providing almost $100 \%$ quantum yield. The largest impact of photosynthesis on life is due to green plants and certain algae in whose RCs a reversible catalytic ET photocycle occurs for which water serves as electron donor. Carbon dioxide is fixed in the form of carbohydrates, and oxygen gas is released as a by-product thereby stabilizing the composition of the Earth's atmosphere.

Three billion years before green plants evolved, photosynthetic energy conversion could be achieved by certain bacteria, for instance the purple bacterium Rhodobacter (Rb.) sphaeroides. These early photosynthetic organisms are simple, 
one-cellular protein-bound donor-acceptor complexes that contain only one RC for light-induced charge-separation. They cannot split water, but rather use hydrogen sulfide or organic compounds as electron donors to reduce $\mathrm{CO}_{2}$ to carbohydrates with the help of sunlight and bacteriochlorophyll as biocatalyst.

In Fig. 1 the structural arrangement of the $\mathrm{RC}$ of $R b$. sphaeroides is shown according to the high-resolution X-ray structure $(3 \AA)$ [11]. The cofactors are embedded in the L, M, H protein domains forming two ET branches, A and B. The $\mathrm{RC}$ of the carotinoid-less strain $\mathrm{R} 26$ of $R b$. sphaeroides contains nine cofactors: the primary donor $\mathrm{P}_{865}$ "special pair" (a bacteriochlorophyll a (BChl) dimer), two accessory BChls $\left(\mathrm{B}_{\mathrm{A}}, \mathrm{B}_{\mathrm{B}}\right)$, two bacteriopheophytins $a\left(\mathrm{BPhe}: \mathrm{H}_{\mathrm{A}}, \mathrm{H}_{\mathrm{B}}\right)$, two ubiquinones $\left(\mathrm{Q}_{\mathrm{A}}, \mathrm{Q}_{\mathrm{B}}\right)$, one non-heme iron $\left(\mathrm{Fe}^{2+}\right)$.

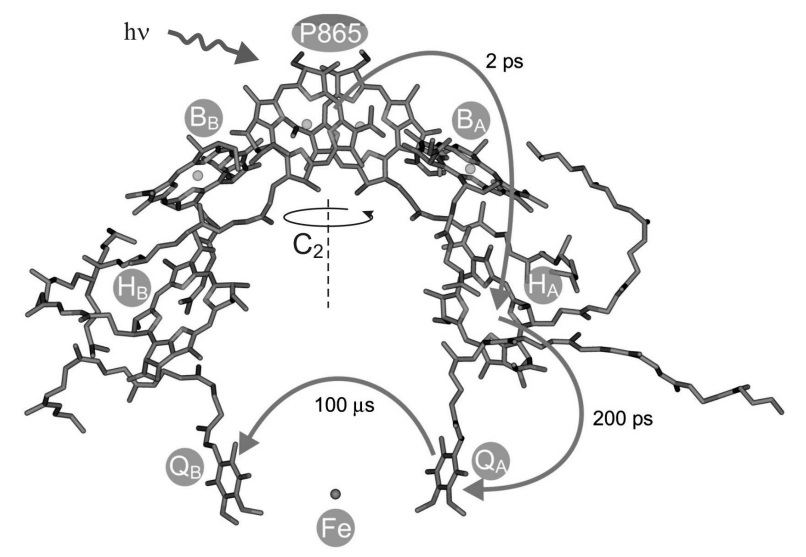

Fig. 1. X-ray structural model of the RC from Rb. Sphaeroides [11] with the protein subunits and the cofactors $\mathrm{P}_{865}, \mathrm{~B}, \mathrm{H}, \mathrm{Q}$, and Fe. Light-induced electron transfer proceeds predominantly along the A branch of the cofactors ("unidirectionality" enigma) despite the approximate $C_{2}$ symmetry of the cofactor arrangement. The ET time constants range from 2 ps to $100 \mu \mathrm{s}$ in the cascade of transmembrane.

As a dominant motif in the evolution of photosynthetic bacteria, an approximate $C_{2}$ symmetry prevails of the cofactor arrangement in the RC. It is intriguing that, despite the apparent two-fold local symmetry of the cofactor arrangement, the primary ET pathway is one-sided along the A branch, as indicated by the arrows in Fig. 1. The origin of this "unidirectionality" enigma of bacterial ET is not yet fully understood despite the numerous elaborate studies, both experimentally and theoretically, performed over the last 10 years. The high-resolution $\mathrm{X}$-ray structure reveals that $C_{2}$ symmetry does not hold for the protein environment of the cofactors, but is broken by different amino acids along the two ET branches. Thereby, the relative energetics and H-bond properties of the cofactors along the two branches will be different. They control the participation of cofactors as intermediate states in the ET cascade. 
It has been only recently demonstrated that specific double-site mutations in the vicinity of the primary donor and an accessory BChl can significantly change the partition of ET between the A and B branches [12, 13]. This is a strong indication that in the wild-type system the breakage of symmetry in the ET pathways is largely due to the finely tuned energetics and electronic couplings of the primary donor and the intermediary acceptors. The unidirectional nature of the primary ET route is probably not determined by a single structural feature, but rather by the concerted effects of small contributions of several different optimized factors. Both the wave functions and the energetics of the cofactors involved can be systematically changed by selectively exchanging neighbouring amino-acid residues of the protein environment by means of site-specific mutation. This can be accomplished, for instance, by introducing or disrupting H-bonds of the cofactors or by changing the ligation of the $\mathrm{Mg}$ in the chlorophyll macrocycles of $\mathrm{P}$. In this context, it is particularly interesting to systematically study the influence of the environment of the primary donor $\mathrm{P}$, since $\mathrm{P}$ is generally considered to play a key role in the origin of the unidirectionality of the primary charge-separation steps. The effect on the electronic structure caused by such mutations can be measured, for example via characteristic shifts of $g$-tensor and hyperfine-tensor components measured by high-field EPR [14-16] and ENDOR [17-21], respectively.

To gain further insight into the origins and consequences of this asymmetry in the electronic structure, various site-directed mutants of the RC are being investigated in our laboratory by $360 \mathrm{GHz}$ high-field EPR. In some of these mutants the ligands to the magnesium of the bacteriochlorophylls were altered. Figure 2 shows the exchange of the histidine His(M202) by leucine (L) or glutamic acid (E) to generate the mutants HL(M202) and HE(M202), respectively. In the symmetry-related heterodimer mutants HL(M202) and HL(L173) [18], the His that ligates the $\mathrm{Mg}$ in the $\mathrm{BChl}$ is exchanged for a Leu that does not ligate to the metal centre, resulting in a BChl:BPhe heterodimer [11]. In HL(M202), the unpaired electron in $\mathrm{P}^{+} \bullet$ was shown to reside on the $\mathrm{BChl}(\mathrm{L})[17,18]$, in $\mathrm{HL}(\mathrm{L} 173)$ on the $\mathrm{BChl}(\mathrm{M})$ dimer half [18]. A promising approach to obtain information on the symmetry properties of the electronic structure is offered by $g$-tensor measurements since the $g$-tensor represents a global probe of the electronic wave function of the unpaired electron. $\mathrm{P}^{+} \bullet$ in photosynthetic RCs exhibits extremely small $g$-tensor anisotropies of $\sim 10^{-3}$. It is, therefore, a prime example of a situation where even high-field EPR at $3.4 \mathrm{~T} / 95 \mathrm{GHz}$ (W-band) can only partially resolve the powder pattern of the isotropically disordered samples [14, 15, 22-24].

The further increase of EPR frequency and field to $360 \mathrm{GHz}$ and $12.9 \mathrm{~T}$, however, provides the spectral resolution necessary to both fully resolve all three principal $g$-tensor components of $\mathrm{P}^{+\bullet}$ randomly oriented in frozen solution [25] and to measure their mutation-induced shifts [16] with a high precision. Figure 2 shows the $360 \mathrm{GHz}$ EPR spectra of $\mathrm{P}^{+} \bullet$ of $\mathrm{R} 26$ in comparison with those of the HL(M202) and HE(M202) mutants. Minimum least-squares fits to a model spin 

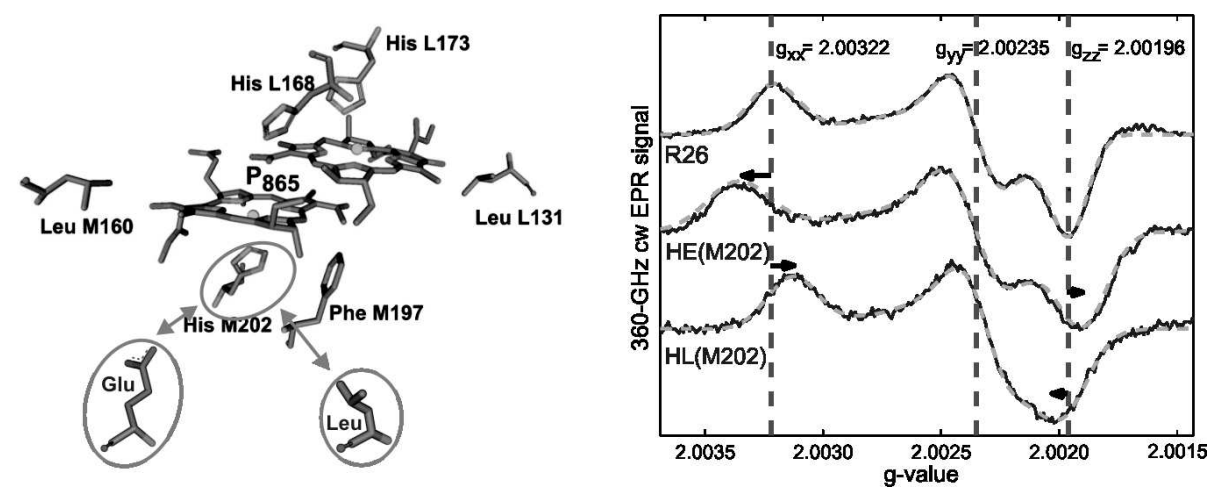

Fig. 2. X-ray structure of the primary-donor special pair $\mathrm{P}_{865}$ and its immediate amino-acid environment in RCs from Rb. Sphaeroides [11]. In the site-directed mutants HL (M202) and HE (M202) the histidine His M202 is replaced by a leucine and by a glutamic acid, respectively. On the right hand side $360 \mathrm{GHz}$ cw EPR spectra of $\mathrm{P}^{+} \bullet$ in RCs from $R b$. sphaeroides mutants at $160 \mathrm{~K}$ are depicted. The most prominent shifts of the $g$-tensor components $g_{x x}$ and $g_{z z}$ due to the mutation at M202 are indicated with solid arrows. Minimum least-squares fits for each spectrum are overlaid with dashed lines (see Ref. [16]).

Hamiltonian including only the Zeeman interaction of radicals with an isotropic orientation distribution are overlaid as dashed lines over the measured spectra. The $g$-values obtained from these fits and the small shifts in the order of $10^{-4}$, that are induced by the mutations of the protein environment of $\mathrm{P}^{+\bullet}$, can be detected with high significance (see Ref. [16]).

The $g$-component shifts observed in our $360 \mathrm{GHz}$ EPR experiments are very surprising: While for $\mathrm{P}^{+} \bullet$ of $\mathrm{HL}(\mathrm{M} 202)$, the overall $g$-tensor anisotropy $\Delta g=\left(g_{x x}-g_{z z}\right)$ becomes smaller (more like that of the monomer), for HE(M202) $\Delta g$ increases to a value considerably larger than for R26.

This unexpected behaviour of $\Delta g$ strongly suggests a local structural change of $\mathrm{BChl}(\mathrm{L})$ as a consequence of the M202 ligand mutations. This conclusion is also supported by the observation of considerable rearrangements in the spin density distributions on $\mathrm{BChl}(\mathrm{L})$ in the two mutants, as was shown previously by previous ENDOR/TRIPLE studies [26]. This observation urged us to perform theoretical calculations of the $g$-tensors of the three $\mathrm{P}^{+} \bullet$ species as a function of different torsional angles of the acetyl group at $\mathrm{BChl}(\mathrm{L})$. We have applied advanced relativistic density functional theory (DFT) methods for the calculation of magnetic resonance parameters (see Ref. [16]). The essential conclusion from the current computational results is that our model predicts a range of increasing torsional angles between $0^{\circ}$ and $143.35^{\circ}$ in the two mutants, and the calculated shifts $\Delta g$ are qualitatively in accordance with our experimental results. 


\subsection{Bacteriorhodopsin}

Nature has invented photosynthesis twice, i.e., the strategy to use sunlight as an energy source to synthesize adenosine triphosphate (ATP): In the photosynthetic reaction centre protein complex of purple bacteria this is initiated by light-induced primary electron transfer between bacteriochlorophyll and quinone cofactors, mediated by the protein microenvironment. In the bacteriorhodopsin protein complex this is set going by light-initiated primary proton transfer between amino-acid residues, mediated by conformational changes of the only cofactor, the retinal.

$\mathrm{BR}$ is a $26 \mathrm{kDa}$ protein complex located in the cell membrane of halophilic archer-bacteria such as Halobacterium salinarium. High-resolution (1.6 ̊) X-ray crystallography coordinates are available for the ground state structure [27] (see Fig. 3). Seven transmembrane helices (A-G) enclose the chromophore retinal which is covalently attached to the amino-acid lysine, K216, on helix G via a protonated Schiff base. Absorption of $570 \mathrm{~nm}$ photons initiates the all trans to 13 cis photoisomerization of the retinal. The Schiff base then releases a proton to the extracellular medium and is subsequently reprotonated from the cytoplasm. Transient intermediates of this catalytic photocycle can be distinguished by the different absorption properties of the retinal, and a sequence of intermediates J, K, L, M, $\mathrm{N}$, and $\mathrm{O}$ has been characterized by time-resolved absorption spectroscopy [28]. Double-flash experiments revealed that the $\mathrm{M}$ intermediate is divided into two substates, $\mathrm{M}_{1}$ and $\mathrm{M}_{2}[29,30]$. During this photocycle conformational changes
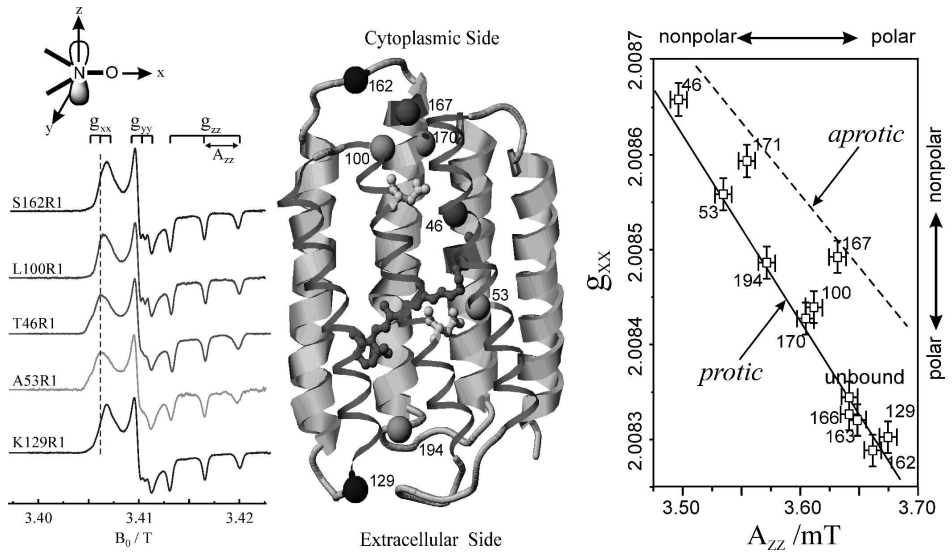

Fig. 3. Left: Experimental W-band cw EPR spectra for a set of BR mutants spin-labelled with the nitroxide side-chain (R1). Middle: Structural model of BR. The $\mathrm{C}_{\alpha}$ atom of the spin-labelled residues, the chromophore retinal, and D96 and D85 participating in the $\mathrm{H}^{+}$transfer are indicated. Right: Plot of $g_{x x}$ vs. $A_{z z}$ of the nitroxide side-chains for various spin-label positions in BR (see the text). The "protic" and "aprotic" limiting cases are placed with reference to the theory, see Ref. [36]. 
of the protein (and the retinal) occur, as has been detected by a variety of experimental techniques (for a review see, e.g., Ref. [31]). The physiological role of such changes is discussed to ensure that release and uptake of protons do not occur from the same side of the membrane, but rather enable BR to work as a vectorial transmembrane proton pump. To this end, in wild-type BR conformational changes associated with the M1 to M2 transition are suggested to function as a "reprotonation switch" required for the vectorial proton transport. Hence, it is believed that during the lifetime of the M state the accessibility of the Schiff base for protons is switched from the extracellular to the cytoplasmic side of the membrane. Detailed analyses of the nature of the conformational changes include neutron diffraction, electron microscopy, X-ray diffraction, solid-state NMR or EPR spectroscopy. They reveal the major changes to be localized at the cytoplasmic moieties of helices $\mathrm{F}$ and $\mathrm{G}$. These helix movements in wild-type BR have been shown to provide an "opening" of the protein to protons on the cytoplasmic end of the transmembrane proton channel [32] and, thus, should allow proton transfer to occur from the internal aspartic-acid proton donor, D96, to the Schiff base during the $\mathrm{M}$ to $\mathrm{N}$ transition. The reprotonation of $\mathrm{D} 96$ from the cytoplasm occurs during the recovery of the BR initial state. A detailed inspection of the structure of the unilluminated state of the protein reveals that certain amino-acid side chains block the proton pathway from the cytoplasm to D96 [32]. The region between D96 and the Schiff base is largely nonpolar, packed with bulky amino-acid residues. Hence, in this unilluminated state the Schiff base is effectively inaccessible to protons from the cytoplasm. In the light-driven $\mathrm{M}_{1}$ to $\mathrm{M}_{2}$ transition, this region is opened for access of protons to the Schiff-base nitrogen atom. However, the question remains whether the large conformational changes observed in the photocycle of the wild-type and many BR mutants are a prerequisite for vectorial proton transport, i.e., really represent the proposed reprotonation switch.

Obviously, additional spectroscopic experiments are needed on wild-type and strategically constructed BR mutants to follow also small conformational changes of protein and cofactor during the photocycle. In this respect the combination of site-directed mutagenesis for spin labelling and high-field EPR for resolving structural changes of proteins at work is particularly powerful.

\subsubsection{Site-directed spin labelling}

During the photocycle of BR no paramagnetic intermediates occur, i.e., neither radicals, radical pairs nor triplet states. To enable the application of EPR, doublet-state spin labels $(S=1 / 2)$ have to be introduced to the protein (in contrast to NMR which probes $S=0$ (singlet states)). The site-directed spin-labelling (SDSL) technique in combination with X-band EPR spectroscopy was pioneered by Hubbell et al. [33]. The recent extension to SDSL/high-field EPR has opened new perspectives for studying structure and dynamics of large NO•-labelled proteins during biological action [9, 34-37]. Figure 4 demonstrates 


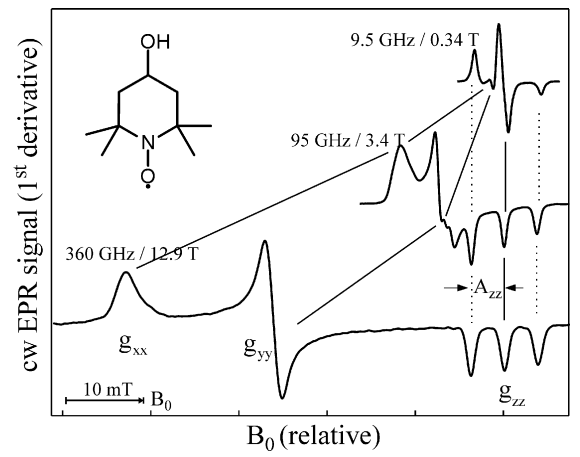

Fig. 4. $\mathrm{Cw}$ EPR spectra of a nitroxide radical (OH-TEMPO) in frozen water solution at different microwave frequency $/ B_{0}$ settings [38]. The spectra are plotted relative to the fixed $g_{z z}$ value.

the remarkable gain in resolution of nitroxide-radical spectra, i.e., the separation of the $g_{x x}, g_{y y}, g_{z z}$ components in relation to the $A_{z z}$ hyperfine splitting, when increasing the Zeeman field from X-band EPR to $95 \mathrm{GHz}$ and $360 \mathrm{GHz}$ EPR. The SDSL technique requires selective cysteine-substitution mutagenesis of the protein with subsequent modification of the unique sulfhydryl group of cysteine with a nitroxide reagent, for example (1-oxil-2,2,5,5-tetramethylpyrroline-3-methyl)methanethiosulfonate, commonly abbreviated as MTS spin label. For systematic studies, a set of SDSL mutants is constructed, each containing a single nitroxide-containing amino-acid side chain, differing by position in the protein sequence. The photocycle of all spin-labelled mutants was checked to ensure that the overall function of the BR protein is retained [9, 34, 35].

\subsubsection{Hydrophobic barrier of the BR proton-transfer channel}

By $95 \mathrm{GHz}$ (W-band) high-field EPR details of the polarity profile along the putative proton channel were probed by $g$ - and hyperfine-tensor components from a series of 10 site-specifically nitroxide spin-labelled BR mutants, with MTS spin label as the reporter side chain R1 [34]. Previous studies of a large number of spin-labelled proteins have shown that the $A_{z z}$ component of the hyperfine tensor and the $g_{x x}$ component of the $g$-tensor are particularly sensitive probes of the microenvironment of the $\mathrm{NO}^{\bullet}$ side chain R1. They allow one to measure changes in polarity and proticity, i.e., $g_{x x}$ and $A_{z z}$ probe the local electric fields and the availability of $\mathrm{H}$-bond forming partners of nearby amino-acid residues or water molecules [34-36]. Moreover, the dynamic properties of the $\mathrm{NO}^{\bullet}$ side chain and, thus, the EPR spectral lineshape have been shown to contain direct information about constraints to motion that are introduced by the secondary and tertiary structures of the protein in the vicinity of the nitroxide binding site [34, 35].

For measuring the polarity changes, W-band EPR spectra were recorded at temperatures below $200 \mathrm{~K}$ to avoid motional averaging of the anisotropic magnetic 
tensors. At these temperatures, R1 can be considered as immobilized on the EPR time scale. The spectra of selected mutants are shown in Fig. 3. They exhibit the typical nitroxide powder-pattern lineshape expected for an isotropic distribution of diluted radicals. The variations of $g_{x x}$ and $A_{z z}$ with the nitroxide binding site can be measured with high precision and plots of $g_{x x}$ and $A_{z z}$ vs. R1 position along the proton channel directly reflect the hydrophobic barrier which the proton has to overcome on its way through the protein channel.

The analysis of both tensor components, $g_{x x}$ and $A_{z z}$, allows one to characterize the $\mathrm{R} 1$ environment in terms of protic and aprotic surroundings. Theoretically, both $g_{x x}$ and $A_{z z}$ are expected to be linearly dependent on the $\pi$-spin density $\rho^{0}$ at the oxygen atom of the nitroxide group. For $g_{x x}$, however, apart from a direct proportionality to $\rho^{0}$, there is an additional dependence on specific properties of the oxygen lone-pair orbitals. The lone-pair orbital energy $E_{n}$ affects $g_{x x}$ via the excitation energy $\Delta E_{n \pi^{*}}=E_{\pi^{*}}-E_{n}$ and is known to be sensitive to the polarity of the environment. It is particularly sensitive to H-bonding of the lone pairs to water or to polar amino-acid residues. Thus, the plot of $g_{x x}$ vs. $A_{z z}$ should indicate the presence or absence of H-bonds in the spin-label environment, i.e., its proticity. This dependence is plotted in Fig. 3 for various spin-label positions in BR [34]. Obviously, two straight-line correlations can be deduced. The points corresponding to positions 46,171 , and 167 belong to a line whose slope is different from that for the remaining points. These three positions can be classified to be exposed to an aprotic environment [39, 40], the other ones to a protic environment. This allows one to characterize the hydrophobic barrier of the BR proton channel in terms of different accessibilities of the respective protein regions to water molecules. The sensitivity of the resolved $g_{x x}$ and $A_{z z}$ tensor components of nitroxide spin labels to the polarity of their micro-environment was recognized also by other high-field EPR research groups, for example in frozen solution [41] and phospholipid membranes [37, 42, 43].

\subsubsection{Conformational changes during the BR photocycle}

The BR triple mutant D96G/F171C/F219L reveals a remarkable conformation of the dark state: It has been shown recently to resemble that of the late $\mathrm{M}$ intermediate (preceding $\mathrm{N}$ in the photocycle) in wild-type BR with a conformation that is retained upon illumination [32, 35, 44, 45]. The triple mutant was spin-labelled and studied by W-band high-field EPR without and with light irradiation [35]. The goal was to test the sensitivity of selectively spin-labelled helix segments in singly, doubly, and triply mutated BR towards changes of the microenvironment in the cytoplasmic proton entrance region during the photocycle. In these studies, we chose position 171 at the cytoplasmic end of helix $\mathrm{F}$ in the single mutant F171C, in the double mutant D96G/F171C, and in the triple mutant D96G/F171C/F219L and attached an MTS spin label to the unique cysteine to form the side chain $\mathrm{R} 1$. The $\mathrm{R} 1$ probe is used to measure the polarity changes 
in this region via light-induced shifts of the $g_{x x}$ and $A_{z z}$ tensor components. The results nicely show that upon light excitation of the single mutant to its $\mathrm{M}$ state, the $\mathrm{NO} \bullet$ residue at position 171 experiences the same non-polar microenvironment as the triple mutant with its pseudo-M state open for proton uptake already in the dark [35].

Pronounced conformational changes of wild-type BR during the photocycle were also observed in the high-field EPR spectra of selectively spin-labelled double mutants V167R1/D96N and V101R1/D96N in their ground states recorded in the dark, and under light illumination. The dark-minus-light spectra clearly show an increased reorientation mobility of the nitroxide side-chain R1 in the M intermediate of the V161R1/D96N mutant, but a decreased mobility for V101R1/D96N. Obviously, the residual anisotropy of the nitroxide motion changes during the photocycle owing to changing space restrictions within the binding site. Label V167R1 is located at the cytoplasmatic moiety of helix F and oriented towards helix C. Thus, an increase of the interhelical distance, caused by motion of helix F or helix C, would account for the experimental data - in accordance with neutron-diffraction and FT-IR results (for details see [34]).

\subsection{Colicin A bacterial toxin}

Colicin A is a member of a family of bacterial toxins that form transmembrane ion channels [46-48]. They are water-soluble proteins, mostly about $70 \mathrm{kD}$ in size, and have varying homology among themselves. Colicin A kills unprotected cells of attacked organisms by inserting specific portions of protein sub-domains into the cytoplasmic membrane forming a voltage-gated ion channel. The open channel leads to electrical depolarization of the membrane and depletion of intracellular ion pools, which ultimately leads to cell death. To understand these mechanisms on the molecular level is currently also of biomedical interest, since insertion of proteins into membranes and subsequent channel formation are common to many toxic proteins of bacterial pathogens found in organisms ranging from bacteria to humans, such as the diphtheria, tetanus, and cholera toxins [47-51].

The colicin toxins have to overcome the protecting barriers of the attacked cell $[46,52]$. Accordingly, Colicin A consists of three functional protein domains: the central receptor domain, $\mathrm{R}$, the $\mathrm{N}$-terminal translocation domain, $\mathrm{T}$, to penetrate the outer membrane and to traverse the periplasm, and the C-terminal channel-forming domain, $\mathrm{C}$, to penetrate the inner membrane which protects the cytoplasm of the cell $[48,53]$. These distinct functions of the colicins are reflected by their three-dimensional shape. The X-ray crystal structure of a complete ( $\mathrm{T}-\mathrm{R}-\mathrm{C})$ Colicin Ia protein was recently determined to $3.0 \AA$ resolution [54] now refined to $2.3 \AA$ [48], and reveals a harpoon-shaped molecule, $210 \AA$ long, with the three-functional domains well separated from each other thereby overcoming the protection barriers of the attacked cell.

The isolated C-domain retains its channel-forming ability in aqueous solutions of artificial membranes $[55,56]$, such as lipid vesicles. Hence, details of 
refolding processes of the C-domain can be studied by in vitro experiments (see below). The X-ray crystal structure of the 204 residue $(21 \mathrm{kD})$ channel-forming C-domain of Colicin A in its water-soluble conformation is available to $2.4 \AA$ resolution [57]. Ten $\alpha$-helices, eight amphiphilic, two hydrophobic are arranged in such a way that the amphiphilic helices surround the hydrophobic hairpin (helices 8 and 9) deeply buried in the centre of the protein. Thereby the 8,9 hairpin is shielded from the contact with the water solvent.

In view of the difficulties encountered with determining X-ray structures of transient proteins in action, numerous spectroscopic techniques are being used to study colicins on the molecular level. Despite many years of work including EPR $[52,58,59]$ the details of the refolding processes upon membrane association and channel formation are not yet known. Since Colicin A is now so well characterized, it may serve as a paradigm system to answer an intriguing question of general interest in molecular biology and medicine: What is the mechanism to switch on the insertion of water-soluble pore-forming proteins into the nonpolar lipid environment of a membrane?

\subsubsection{Models of transmembrane ion-channel formation}

The C-domain of Colicin A (and other members of the colicin family) can adopt two conformations, the water-soluble form and the transmembrane form. The transition between these conformations requires massive refolding of the tertiary structure. To date, two alternative models are being discussed to explain how the C-domain turns itself inside out to form the membrane-associated state with pore formation, the "umbrella" model [57] and the "penknife" model [60] (see Fig. 5). Conceptually, they differ in the description of the relatively slow (100 ms-s range) membrane-insertion step to be either spontaneous or voltage dependent. After docking to the membrane surface, a slow, still voltage-independent,

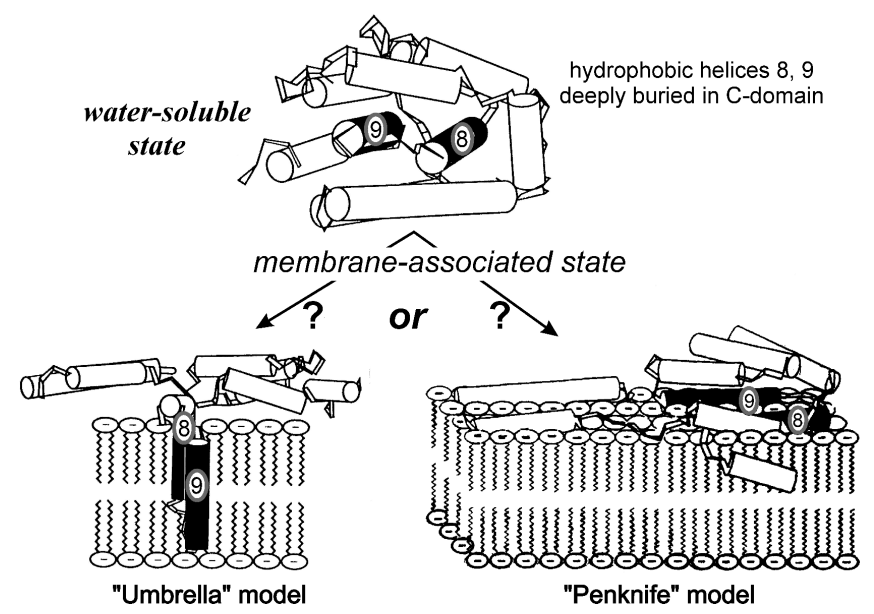

Fig. 5. The "umbrella" model [57] and the "penknife" model [60] of the membraneassociated state of the channel-forming C-domain of Colicin A. 
refolding of the C-domain occurs to bring the hydrophobic helices 8 and 9 to the outside of the protein complex. In the umbrella model the hydrophobic hairpin 8, 9 spontaneously traverses the membrane, whereas in the penknife model the refolding leaves the 8,9 hairpin close to the membrane surface, but a change of the electric transmembrane potential is required to trigger insertion of the 8,9 hairpin into the membrane and, ultimately, to open the channel for ion flow.

\subsection{2. $95 \mathrm{GHz} E P R$ on membrane-insertion mechanisms}

In our work on Colicin A transient states we applied high-field EPR at $95 \mathrm{GHz}$ in conjunction with site-directed spin-labelling techniques using MTS as the nitroxide spin label [9]. Owing to the high spectral resolution achieved by $95 \mathrm{GHz}$ EPR we could use both the $A_{z z}$ hyperfine-tensor component of the ${ }^{14} \mathrm{~N}$ nucleus and the $g_{x x}$ tensor component as sensitive probes for the polarity and proticity of the microenvironment of the nitroxide side chain R1 and for its motional characteristics in three-dimensional space under the local constraints of the protein [34-36].

In Fig. 6, by numbered spheres the five individual amino-acid residues of helix 9 of the C-domain are indicated, which have been replaced by cysteines via exchange mutagenesis $[60,61]$ at residue positions $169,176,181,183$, and 184, respectively. The single cysteines were spin labelled with the nitroxide label MTS providing a nitroxide side chain R1. Our experimental strategy for detecting the
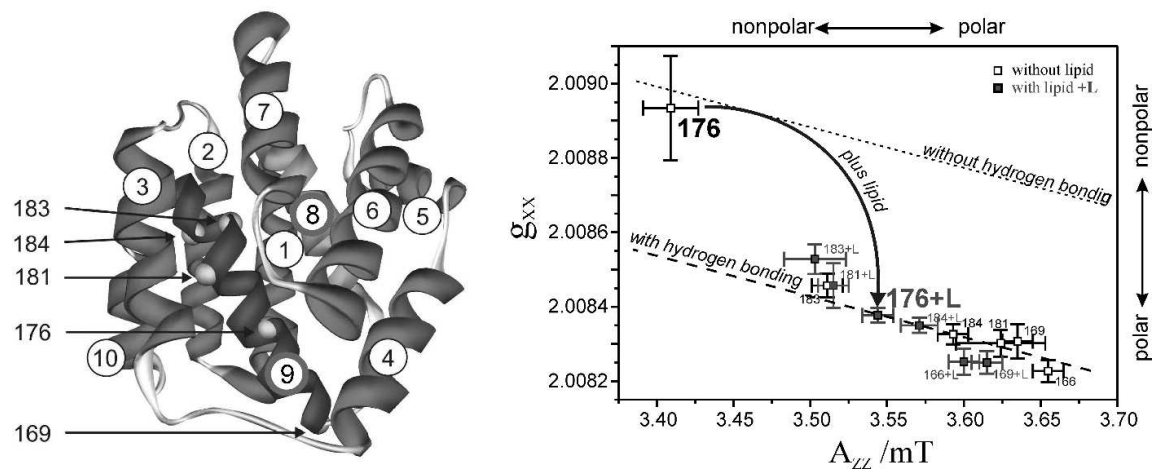

Fig. 6. Left: X-ray structure of the channel-forming C-domain of Colicin A. The positions of cysteine replacements in helix 9 by site-directed exchange mutagenesis are indicated by numbered spheres $(169,176,181,183,184)$. The $\alpha$-helices are labelled 1 (N-terminus) to 10 (C-terminus). The hydrophobic helices are 8 and 9 . For details, see Refs. [57, 60, 61]. Right: Polarity plot of $g_{x x}$ vs. $A_{z z}$ for the various nitroxide spin-label positions in helix 9 of site-directed Colicin A mutants. The measured tensor components with addition of lipid vesicles and without lipid addition are marked by full and open squares, respectively. The dashed lines define the limits between the non-hydrogen bonded (short dashes) and the fully hydrogen bonded (long dashes) cases. For details, see Refs. [9, 34, 36]. 
refolding of the $\mathrm{C}$-domain under membrane association was to compare the EPR spectra of Colicin A in buffered aqueous solution under physiological conditions ( $\mathrm{pH} 8)$ with those after adding lipids to the sample to form vesicles as artificial membranes [9]. To this end, small unilamellar vesicles were made by sonification of DMPG (1,2-dimyristoyl-sn-glycero-3-phospho-rac-(1-glycerol)) in water.

The ${ }^{14} \mathrm{~N}$ hyperfine-tensor components $A_{z z}$ and the $g_{x x}$ tensor components were measured for the five mutants. In the water-soluble conformation (no lipid added) the $A_{z z}$ and $g_{x x}$ values reveal high polarity at both ends of helix 9 (positions 184, 169), and a lower polarity in the centre (position 176). This finding is consistent with the amino-acid arrangement according to the X-ray crystal structure [57]. After addition of lipid a rather uniform, high-polarity character of the nitroxide microenvironment of all mutants results. The highest polarity change is experienced by the $\mathrm{NO}^{\bullet}$ side chain attached to position 176 in the central part of the helix.

In Fig. 3 the W-band measurements of $g_{x x}$ and $A_{z z}$ are summarized. Both probes show that the central-region position 176 experiences a strong change in polarity of the microenvironment towards more polar character, whereas in the vicinity of the end-region positions 169, 184 only weak polarity changes occur upon adding lipids. This different behaviour of polarity changes upon membrane association for position 176 and positions 169, 184 is especially evident from this plot $g_{x x}$ vs. $A_{z z}$.

In the case that the umbrella model is valid, helices 8,9 should penetrate spontaneously the membrane so that their central part, as probed by the nitroxide side chain 176 in helix 9, would be placed into the membrane's interior, i.e., in a highly nonpolar region. This means that in the umbrella model one would expect for spin label 176 no large changes of $g_{x x}$ and $A_{z z}$ upon adding lipid to the aqueous sample because in both states, water-soluble and membrane-associated, the microenvironment would remain nonpolar. In the case that the penknife model is valid, however, helix 9 should remain for some time in a transient state close to the membrane surface - until an electric potential change initiates helix insertion and pore formation in the membrane. In this situation, position 176 would experience a drastic change of the microenvironment from nonpolar to polar in the membrane-associated state. This is exactly what we observe via the polarity probes $g_{x x}$ and $A_{z z}$ when adding vesicle-forming lipids to the aqueous sample. Hence, our data are not consistent with the umbrella model, but validate the penknife model for membrane association of the Colicin A channel-forming domain. This conclusion is in agreement with experiments on Colicin A double mutants with site-specifically attached fluorescence labels described in Ref. [60] (for more details, see [9]).

\section{Conclusions and outlook}

In this overview it is shown that modern multifrequency EPR spectroscopy at high magnetic fields provides detailed information about structure and dynam- 
ics of transient radicals and radical pairs occurring in biological electron- and ion-transfer processes. Thereby our understanding of the relation between structure, dynamics, and function of molecular switches is considerably improved. This holds with respect to the fine-tuning of electronic properties of donor and acceptor cofactors by means of weak interactions with their protein and lipid environment, such as H-bonding to specific amino-acid residues. This also holds with respect to the massive protein refolding in the course of transmembrane ion-channel formation.

As summarising conclusions relevant to biological systems it is pointed out that:

- by high-field/high-frequency EPR even on disordered samples orientation-selective hydrogen bonding and polar interactions in the protein binding sites can be traced. This is important information complementary to what is available from high-resolution X-ray diffraction of protein single crystals;

- in electron-transfer processes often several organic radical species are generated as transient intermediates. To distinguish them by the small differences in their $g$-factor and hyperfine interactions, high-field/high-frequency EPR becomes the method of choice;

- high-field/high-frequency cw EPR generally provides, by lineshape analysis, shorter time windows down into the ps range for studying correlation times and fluctuating local fields over a wide temperature range. They are associated with characteristic dynamic processes, such as protein, cofactor or lipid motion and protein folding;

- pulsed high-field/high-frequency EPR provides real-time access to specific cofactor and/or protein motions in the ns time scale. Motional anisotropy can be resolved which is governed by anisotropic interactions, such as hydrogen bonding along specific molecular axes within the binding site;

- ENDOR at high Zeeman fields takes additional advantage of the orientation selection of molecular sub-ensembles in powder or frozen-solution samples. Thereby, even in the case of small $g$-anisotropies, ENDOR on cofactors can provide single-crystal like information about hyperfine interactions, including anisotropic hydrogen bonding to the protein.

Hence, high-field EPR adds substantially to the capability of "classical" spectroscopic and diffraction techniques for determining structure-dynamics-function relations of biosystems, since transient intermediates can be observed in real time while they are staying in their working states at biologically relevant time scales.

\section{Acknowledgments}

Numerous coworkers — students, postdocs, colleagues — from different parts of the world have contributed to our interdisciplinary high-field EPR work, to all of them we want to express our gratitude. Financial support by the Deutsche 
Forschungsgemeinschaft (in the frame of the DFG priority programs SPP 1051, SFB 498) is gratefully acknowledged.

\section{References}

[1] J.H. Freed, Annu. Rev. Phys. Chem. 51, 65 (2000).

[2] A. Schweiger, G. Jeschke, Principles of Pulse Electron Paramagnetic Resonance, University Press, Oxford 2001.

[3] G. Rist, J.S. Hyde, J. Chem. Phys. 52, 4633 (1970).

[4] M. Rohrer, F. MacMillan, T.F. Prisner, A.T. Gardiner, K. Möbius, W. Lubitz, J. Phys. Chem. B 102, 4648 (1998).

[5] Y.S. Lebedev, in: Modern Pulsed and Continuous-Wave Electron Spin Resonance, Eds. L. Kevan, M.K. Bowman, John Wiley, New York 1990, p. 365.

[6] K. Möbius, A. Savitsky, M. Fuchs, in: Very High Frequency (VHF)ESR/EPR, Eds. O. Grinberg, L.J. Berliner, Vol. 22, Kluwer/Plenum Publishers, New York 2004, p. 45.

[7] O. Burghaus, M. Rohrer, T. Götzinger, M. Plato, K. Möbius, Meas. Sci. Technol. 3, 765 (1992).

[8] T.F. Prisner, M. Rohrer, K. Möbius, Appl. Magn. Reson. 7, 167 (1994).

[9] A. Savitsky, M. Kühn, D. Duché, K. Möbius, H.J. Steinhoff, J. Phys. Chem. B 108, 9541 (2004).

[10] M.R. Fuchs, T.F. Prisner, K. Möbius, Rev. Sci. Instr. 70, 3681 (1999).

[11] A.J. Chirino, E.J. Lous, M. Huber, J.P. Allen, C.C. Schenck, M.L. Paddock, G. Feher, D.C. Rees, Biochemistry 33, 4584 (1994).

[12] A.L.M. Haffa, S. Lin, J.C. Williams, A.K.W. Taguchi, J.P. Allen, N.W. Woodbury, J. Phys. Chem. B 107, 12503 (2003).

[13] A.L.M. Haffa, S. Lin, J.C. Williams, B.P. Bowen, A.K.W. Taguchi, J.P. Allen, N.W. Woodbury, J. Phys. Chem. B 108, 4 (2004).

[14] R. Klette, J.T. Törring, M. Plato, K. Möbius, B. Bönigk, W. Lubitz, J. Phys. Chem. 97, 2015 (1993).

[15] M. Huber, J.T. Törring, Chem. Phys. 194, 379 (1995).

[16] M.R. Fuchs, A. Schnegg, M. Plato, C. Schulz, F. Müh, W. Lubitz, K. Möbius, Chem. Phys. 294, 371 (2003).

[17] J. Rautter, F. Lendzian, C. Schulz, A. Fetsch, M. Kuhn, X. Lin, J.C. Williams, J.P. Allen, W. Lubitz, Biochemistry 34, 8130 (1995).

[18] M. Huber, R.A. Isaacson, E.C. Abresch, D. Gaul, C.C. Schenck, G. Feher, Biochim. Biophys. Acta 1273, 108 (1996).

[19] K. Artz, J.C. Williams, J.P. Allen, F. Lendzian, J. Rautter, W. Lubitz, Proc. Nat. Acad. Sci. USA 94, 13582 (1997).

[20] F. Müh, M. Bibikova, F. Lendzian, D. Oesterhelt, W. Lubitz, in: Photosynthesis: Mechanisms and Effects, Ed. G. Garab, Vol. 2, Kluwer: Dordrecht, 1998, p. 763.

[21] F. Müh, F. Lendzian, M. Roy, J.C. Williams, J.P. Allen, W. Lubitz, J. Phys. Chem. B 106, 3226 (2002). 
[22] O. Burghaus, M. Plato, M. Rohrer, K. Möbius, F. MacMillan, W. Lubitz, J. Phys. Chem. 97, 7639 (1993).

[23] W. Wang, R.L. Belford, R.B. Clarkson, P.H. Davis, J. Forrer, M.J. Nilges, M.D. Timken, T. Walczak, M.C. Thurnauer, J.R. Norris, A.L. Morris, Y. Zhang, Appl. Magn. Reson. 6, 195 (1994).

[24] M. Huber, J.T. Törring, M. Plato, U. Finck, W. Lubitz, R. Feick, C.C. Schenck, K. Möbius, Sol. Energy Mater. Sol. Cells 38, 119 (1995).

[25] P.J. Bratt, E. Ringus, A. Hassan, H.V. Tol, A.-L. Maniero, L.-C. Brunel, M. Rohrer, C. Bubenzer-Hange, H. Scheer, A. Angerhofer, J. Phys. Chem. B 103, 10973 (1999).

[26] C. Schulz, F. Müh, A. Beyer, R. Jordan, E. Schlodder, W. Lubitz, in: Photosynthesis: Mechanisms and Effects, Ed. G. Garab, Kluwer: Vol. 2, Dordrecht 1998, p. 767 .

[27] H. Luecke, B. Schobert, H.-T. Richter, J.-P. Cartailler, J.K. Lanyi, J. Mol. Biol. 291, 899 (1999).

[28] G. Váró, J.K. Lanyi, Biochemistry 30, 5008 (1991).

[29] S. Druckmann, N. Friedmann, J.K. Lanyi, R. Needleman, M. Ottolenghi, M. Shewes, Photochem. Photobiol. 56, 1041 (1992).

[30] B. Hessling, J. Herbst, R. Rammelsberg, K. Gerwert, Biophys. J 73, 2071 (1997).

[31] U. Haupts, J. Tittor, D. Oesterhelt, Annu. Rev. Biophys. Biomol. Struct. 28, 367 (1999).

[32] S. Subramaniam, R. Henderson, Nature 406, 653 (2000).

[33] W.L. Hubbell, C. Altenbach, Curr. Opin. Struct. Biol. 4, 566 (1994).

[34] H.-J. Steinhoff, A. Savitsky, C. Wegener, M. Pfeiffer, M. Plato, K. Möbius, Biochim. Biophys. Acta 1457, 253 (2000).

[35] C. Wegener, A. Savitsky, M. Pfeiffer, K. Möbius, H.-J. Steinhoff, Appl. Magn. Reson. 21, 441 (2001).

[36] M. Plato, H.-J. Steinhoff, C. Wegener, J.T. Törring, A. Savitsky, K. Möbius, Mol. Phys. 100, 3711 (2002).

[37] A.I. Smirnov, in: Electron Paramagnetic Resonance, A Specialist Periodical Report, Eds. B.C. Gilbert, M.J. Davies, K.A. McLauchlan, Vol. 18, Cambridge 2002, p. 109.

[38] T. Kawamura, S. Matsunami, T. Yonezawa. Bull. Chem. Soc. Jpn. 40, 1111 (1967).

[39] O.H. Griffith, P.J. Dehlinger, S.P. Van, J. Membrane Biol. 15, 159 (1974).

[40] M.A. Ondar, O.Y. Grinberg, Y.S. Lebedev, Sov. J. Chem. Phys. 3, 781 (1985).

[41] K.A. Earle, J.K. Moscicki, M. Ge, D.E. Budil, J.H. Freed, Biophys. J 66, 1213 (1994).

[42] D. Marsh, D. Kurad, V.A. Livshits, Chemistry and Physics of Lipids 116, 93 (2002).

[43] S. Subramaniam, I. Lindahl, P. Bullough, A.R. Faruqi, J. Tittor, D. Oesterhelt, L. Brown, J. Lanyi, R. Henderson, J. Mol. Biol. 287, 145 (1999). 
[44] J. Tittor, S. Paula, S. Subramaniam, J. Heberle, R. Henderson, D. Oesterhelt, J. Mol. Biol. 319, 555 (2002).

[45] J.H. Lakey, G.F.v.d. Groot, F. Pattus, Toxicology 87, 85 (1994).

[46] R.M. Stroud, Curr. Opin. Struct. Biol. 5, 514 (1995).

[47] R.M. Stroud, K. Reiling, M. Wiener, D. Freymann, Curr. Opin. Struct. Biol. 8, 525 (1998).

[48] K.J. Oh, H. Zhan, C. Cui, K. Hideg, R.J. Collier, W.L. Hubbell, Science 273, 810 (1996).

[49] P.D. Huynh, C. Cui, H. Zhan, K.J. Oh, R.J. Collier, A. Finkelstein, J. Gen. Physiol. 110, 229 (1997).

[50] D.B. Lacy, R.C. Stevens, Curr. Opin. Struct. Biol. 8, 778 (1998).

[51] L. Salwinski, W.L. Hubbell, Protein Sci. 8, 562 (1999).

[52] W.A. Cramer, J.B. Heymann, S.L. Schendel, B.N. Deriy, F.S. Cohen, P.A. Elkins, C.V. Stauffacher, Annu. Rev. Biophys. Biomol. Struct. 24, 611 (1995).

[53] M. Wiener, D. Freymann, P. Ghosht, R.M. Stroud, Nature 385, 461 (1997).

[54] J.R. Dankert, Y. Uratani, C. Grabau, W.A. Cramer, M. Hermodson, J. Biol. Chem. 257, 3857 (1982).

[55] A. Nardi, S.L. Slatin, D. Baty, D. Duché, J. Mol. Biol. 307, 1293 (2001).

[56] M.W. Parker, J.P.M. Postma, F. Pattus, A.D. Tucker, D. Tsernoglou, J. Mol. Biol. 224, 639 (1992).

[57] A.P. Todd, J. Cong, F. Levinthal, C. Levinthal, W.L. Hubbell, Proteins 6, 294 (1989).

[58] Y.K. Shin, C. Levinthal, F. Levinthal, W.L. Hubbell, Science 259, 960 (1993).

[59] J.H. Lakey, D. Duché, J.-M. González-Manas, D. Baty, F. Pattus, J. Mol. Biol. 230, 1055 (1993).

[60] D. Duché, M.W. Parker, J.-M. González-Manas, F. Pattus, D. Baty, J. Biol. Chem. 269, 6332 (1994).

[61] M.R. Fuchs. Ph.D. Thesis, Free University Berlin, Berlin 1999. 